\title{
Efecto Regulador de Tibolona sobre la Actividad Antiinflamatoria del Macrófago
}

\section{Regulatory Effect of Tibolone on Antiinflamatory Activity of Macrophage}

\author{
K. A. Aguayo-Cerón', J. A. Morales-González', G. Gutiérrez-Iglesias'1, A. Parra-Barrera', C. A. Jiménez-Zamarripa1,2, \\ M. E. Ocharán-Hernández', C. C. Calzada-Mendoza ${ }^{1}$ \\ IInstituto Politécnico Nacional - Escuela Superior de Medicina \\ 2Hospital Psiquiátrico "Dr. Samuel Ramírez Moreno"
}

\section{RESUMEN}

Los macrófagos son células fagocíticas que activan a la sintasa de óxido nítrico (SON) y la NADPH oxidasa con el objetivo de eliminar agentes que reconocen como extraños. Además, participan en el desarrollo y mantenimiento de la respuesta inflamatoria. Tibolona ( $\mathrm{Tb})$, a través de sus metabolitos, tiene actividad estrogénica, progestagénica y androgénica. Es utilizada como alternativa de la terapia hormonal de la menopausia, que además disminuye marcadores de inflamación. El objetivo de este estudio fue describir el efecto de Tb sobre la expresión de las interleucinas 1, 6, 10 y TNF- $\alpha$ así como la actividad de la NADPH oxidasa del macrófago. Se utilizó la línea celular THP-1, se diferenció a macrófago, y se evaluó la actividad de NADPH oxidasa por el ensayo de NBT y la expresión de IL-1 $\beta$, IL-6, TNF- $\alpha$ e IL-10 por RT-qPCR. Se encontró que Tb regula la actividad enzimática, así como la expresión de citocinas ante un estímulo proinflamatorio. Por lo que se concluye que Tb favorece la actividad antiinflamatoria del macrófago. Estos resultados contribuyen a la descripción de los mecanismos de acción de este fármaco. Además, se propone al macrófago como un blanco de regulación del proceso inflamatorio por acción de Tb. Se sugiere que se determine la expresión proteica de las citocinas por otras técnicas, tales como western blot, ELISA, o citometría de flujo; así como la actividad de SON. 


\section{ABSTRACT}

Macrophages are phagocytic cells that activate NOS and NADPH oxidase to eliminate agents that recognize like strangers, also participate in development and maintain of inflammatory response. In other hand, tibolone (Tb) has three main metabolites, which have an estrogenic, progestogen and androgenic activity. This drug is a hormonal therapy to treat symptoms of menopause, with ability to decrease inflammation markers. The aim of this study is to describe the effect of $\mathrm{Tb}$ on macrophage activity. This study was carried out on differentiated macrophage THP1 cell line used. NADPH oxidase activity by NBT assay and expression of IL-1 $\beta$, IL-6, TNF- $\alpha$ and IL-10 by RT-qPCR were measured. It has been observed that $\mathrm{Tb}$ regulates the enzymatic activity, as well as the cytokines expression in the cells that received a proinflammatory stimulus; these results contribute to description of mechanism of action of this drug. The results suggest that macrophage could be a target for anti-inflammatory action of Tb. Its remain to investigate the protein expression of cytokines and activity of iNOS.

KEYWORDS: macrophage; menopause; tibolone; NADPHoxidase

\section{Correspondencia}

DEStINATARIO: Claudia Camelia Calzada Mendoza

INSTITUCIÓN: Instituto Politécnico Nacional - Escuela

Superior de Medicina

DIRECCIÓN: Plan de San Luis y Díaz Mirón S/N,

Col. Casco de Santo Tomás, Delegación Miguel Hidalgo,

C. P. 11340, Ciudad de México, México

CORREO ELECTRÓNICO: ccalzada@ipn.mx

\section{Fecha de recepción:}

30 de agosto de 2018

Fecha de aceptación:

10 de junio de 2019 


\section{INTRODUCCIÓN}

Los macrófagos son células fagocíticas efectoras de la respuesta inmunológica y entre sus acciones destacan: la eliminación de la infección, erradicación de restos y resorción en tejidos durante la homeostasis, además de participar de manera importante en el inicio, desarrollo y mantenimiento del proceso inflamatorio ${ }^{[1,2]}$.

Estas células poseen una marcada plasticidad que les permite responder a los estímulos del microambiente en el que se encuentren y así modificar su fenotipo ${ }^{[3,4]}$.

Los macrófagos son capaces de ingerir restos celulares o células apoptóticas, así como patógenos, con el objetivo de eliminarlos, para lo cual es necesario que estos sean reconocidos por receptores de membrana. Es así que una vez que son activados a través de los receptores acoplados a proteínas $\mathrm{G}$, inducen la producción de sustancias microbicidas en las que se incluyen a las especies reactivas de oxígeno (ERO) y especies reactivas de nitrógeno (ERN) ${ }^{[2,5,6]}$. Existen numerosas funciones fisiológicas que se controlan por vías de señalización que desencadenan reacciones tipo redox, reguladas principalmente por la sintasa de óxido nítrico (SON) y la NADPH oxidasa.

En estudios in vitro de líneas celulares de macrófagos de ratón, se ha demostrado el efecto del estradiol (E2) para inducir el fenotipo antiinflamatorio de esta célula. Además, en estudios con líneas celulares humanas se ha demostrado que E2 disminuye la actividad del complejo enzimático NADPH oxidasa en el macrófago a través de la activación del receptor expresado en estas células, lo cual contribuye a disminuir el riesgo cardiovascular al prolongar la vida del óxido nítrico (NO) [7].

Tibolona es un fármaco que tiene principalmente tres metabolitos: 3alfa-hidroxi-tibolona ( $3 \alpha-\mathrm{OH}$ ) y 3 beta-hidroxi-tibolona $(3 \beta-\mathrm{OH})$ con actividad estrogénica; y el isómero delta 4-tibolona, con efectos progestagénicos y androgénicos. Es por esa versatilidad de efectos que actualmente se utiliza como una alternativa a la terapia hormonal de la menopausia. Si bien, en un principio este fármaco se prescribía para el tratamiento de la osteoporosis, existen estudios clínicos que han demostrado que en mujeres en posmenopausia con tratamiento con $\mathrm{Tb}$ disminuye los marcadores de la enfermedad cardiovascular ${ }^{[8,9]}$. En diferentes estudios clínicos se ha demostrado que $\mathrm{Tb}$ tiene efectos benéficos sobre el perfil lipídico, al disminuir los niveles de colesterol, triglicéridos y HDL ${ }^{\left[8,{ }^{10]}\right.}$. Además, se ha asociado con la disminución de la incidencia de cáncer ${ }^{[11]}$. También se han reportado que $\mathrm{Tb}$ induce la isoforma endotelial de la SON (SONe), lo cual contribuye con su efecto protector cardiovascular al aumentar las concentraciones de NO en endotelio ${ }^{[12,13]}$.

Asimismo, existen estudios realizados en diferentes líneas celulares que explican los efectos observados en los estudios clínicos, es así como, se ha reportado que $\mathrm{Tb}$ tiene efectos estrogénicos al disminuir la síntesis de moléculas de adhesión en la placa ateromatosa ${ }^{[14]}$. Por otro lado, en líneas celulares de cáncer endometrial, se ha demostrado que $\mathrm{Tb}$ inhibe la proliferación celular a través de su efecto progestagénico ${ }^{[15] .}$

Sin embargo, los estudios no son suficientes para explicar los efectos de Tb en la disminución del proceso inflamatorio. El objetivo de este trabajo fue evaluar el efecto de Tb sobre la actividad de NADPH oxidasa en células THP-1 diferenciadas a macrófagos, así como su efecto en la modulación de la síntesis de algunas citocinas (IL-1 $\beta$, IL-6, IL-10, y TNF- $\alpha$ ).

\section{METODOLOGÍA}

\section{Muestras y reactivos}

Medio modificado de Dulbecco F12 con L-glutamina, 15Mm HEPES, rojo fenol (Caisson Laboratories, EE. UU.); Suero fetal bovino (HyClone, EE. UU.); tibolona, 1-forbol, 12-miristato, 13-acetato (PMA) (Cayman, EE. UU.); dimetilsulfóxido (DMSO); dimetilformamida 
(DMF) (Caledon Laboratories, Canadá); bromuro de 3-(4,5-dimetiltiazol-2-il)-2,5-difeniltetrazolio (MTT); cloruro de tetrazolio p-nitro-azul (NBT), N, N, dimetilformamida (Sigma-aldrich, EE. UU.); alamarBlue (Thermo Fisher Scientific); PBS contiene NaCl (137 $\mathrm{mmol} / \mathrm{l}), \mathrm{KCl}(2,7 \mathrm{mmol} / \mathrm{l})$.

\section{Cultivo y diferenciación celular}

Se cultivaron monocitos THP-1 humanos en medio DMEM/F12 suplementado con suero fetal bovino al $10 \%$ (SFB) en una atmósfera humidificada de 5\% de $\mathrm{CO} 2$ a $37^{\circ} \mathrm{C}$. Se añadió medio fresco cada 3 días. La diferenciación a macrófagos se obtuvo tratando monocitos de THP-1 durante 48 horas con 60 ng/ml de PMA en placas de cultivo celular de 48 pozos con $1 \times 10^{5}$ células en cada uno de ellos. Las células adherentes diferenciadas se lavaron dos veces con medio de cultivo DMEM/F12 sin PMA, pero que contenían 10\% de SFB y se dejaron reposar durante otras $72 \mathrm{~h}$. La diferenciación celular se observó mediante microscopia (microscopio invertido, marca Lauca) a un aumento 40X. Los controles consistieron en: control negativo (Ctrl-), células diferenciadas sin $\mathrm{Tb}$ y sin PMA; control positivo (Ctrl+), células diferenciadas sin fármaco, pero estimuladas con PMA (100 ng/ml); mientras que las condiciones a probar fueron: células preincubadas durante $24 \mathrm{~h}$ con $\mathrm{Tb}$ a una concentración de 10 y $1 \mathrm{uM}$, respectivamente; células co-incubadas durante $24 \mathrm{~h}$ con $\mathrm{Tb}$ a una concentración de 10 y 1 uM respectivamente y que además fueron estimuladas con PMA (100 $\mathrm{ng} / \mathrm{ml}$ ) durante 24h. Para graficar la expresión relativa del mensajero de cada gen, inicialmente se normalizaron con los genes endógenos de cada grupo, donde éstos valores de expresión estuvieron en referencia a la expresión de GAPDH de cada grupo. A estos controles GAPDH se les asignó el valor de 1 para que fueran comparables a la baja o a la alta de las expresiones en todos los tratamientos para cada uno de los genes evaluados. Cabe mencionar que para los controles internos, se desarrolló la reacción sin muestra, la cual fue negativa a la expresión (datos no mostrados).

\section{Ensayo con NBT}

Los macrófagos se cultivaron en placas de 48 pozos ( $1 \times 10^{5}$ por pozo) y se pre-incubaron con Tb durante 24 y 48 h. A continuación, las células se trataron con 500 ng/ml de PMA para activar el sistema enzimático NADPH oxidasas. 24h después de la estimulación anterior, se evaluó la actividad de las oxidasas, añadiendo $100 \mu \mathrm{l}$ de solución de NBT e incubando durante $1.5 \mathrm{~h}$. Al finalizar, las células se lavaron dos veces con PBS, secadas al aire. El NBT precipitado dentro de las células, se disolvió; primero añadiendo $100 \mu \mathrm{l}$ de KOH 2M para solubilizar las membranas celulares, y luego añadiendo $70 \mu$ de DMSO para disolver el formazán azul con agitación suave durante 10 minutos a temperatura ambiente. La solución de NBT obtenida se transfirió a una placa de 96 pozos y se leyó la absorbancia en un lector de microplacas a 620 nm (Whym201, Poweam, lector de microplacas).

\section{Extracción y cuantificación de RNA}

Para la extracción del RNA, las células THP-1, se cultivaron y diferenciaron en una placa de 6 pozos $\left(1 \times 10^{6}\right.$ cel/pozo) y se utilizó el método de tiocinato de guanidina Trizol (Invitrogen, Carlsbad, CA) de acuerdo con las instrucciones del fabricante. Se agregaron $500 \mu \mathrm{lde}$ Trizol a cada pozo (placa de 6) para el lisado de las células y se transfirió a un tubo de $1.5 \mathrm{ml}$, al cual se agregaron $100 \mu$ l de cloroformo, se agitó con el vortex y se dejó incubar a temperatura ambiente de 2 a 3 minutos. Después se centrifugó a $\mathbf{1 2 . 0 0 0}$ rpm por 15 min a $4^{\circ} \mathrm{C}$, la fase acuosa se separó y colocó en un tubo de $1.5 \mathrm{~mL}$, al cual se le agregaron $250 \mu \mathrm{l}$ de isopropanol, se mezcló por inversión y se incubó durante 10 minutos a temperatura ambiente, a continuación, se centrifugó a $12.000 \mathrm{rpm}$ por $10 \mathrm{~min}$ a $4^{\circ} \mathrm{C}$. En el fondo del tubo se observó un botón (RNA) y el sobrenadante fue decantado, se colocaron $500 \mu \mathrm{l}$ de etanol al 75\%, se resuspendió y luego se centrifugó el botón a 12.000 rpm por $10 \mathrm{~min}$ a $4^{\circ} \mathrm{C}$ (dos veces). Finalmente, el RNA se dejó secar durante 1 hora, posteriormente, se resuspendió en agua DEPC. La integridad del RNA se deter- 
minó observando la subunidad 18s y 28s mediante electroforesis en gel de agarosa (2\%), la concentración y la pureza del RNA se cuantificó a través de la medición de la densidad óptica 260/280 (proteínas) y 260/230 (solventes), se consideró adecuado un rango de 1.8-2.2. Nanofotometro NP80 (Implen, Germany).

\section{Expresión génica de citocinas}

Se llevó a cabo la síntesis de cDNA a partir del RNA total (500 ng), utilizando la transcriptasa reversa M-MVL (Thermo Fisher Scientific) de acuerdo a las condiciones del fabricante.

El cDNA obtenido se utilizó para llevar a cabo la amplificación mediante RT-qPCR en el equipo Real Time PCR Pro qPCR 48 (TECHNE, Burlington, NJ), usando SyberGreen como marcador de fluorescencia (FastStar SYBER Green Master, Roche). La reacción se llevó a cabo en un volumen final de $10 \mu$ l, colocando $2.5 \mu$ de buffer de reacción (amortiguador y enzima), $0.3 \mu \mathrm{l}$ de cebadores sentido, $0.3 \mu \mathrm{l}$ de cebadores antisentido, $0.2 \mu \mathrm{l}$ de SyberGreen, $5.7 \mu \mathrm{l}$ de $\mathrm{H}_{2} \mathrm{O}$ grado PCR y $1 \mu \mathrm{l}$ (500 ng) de muestra. Los cebadores usados para la amplificación de los genes fueron los siguientes:

- IL-1 $\beta$, sentido 5'-AGTTGACGGACCCCAAAAG-3' y antisentido 5'-AGCTGGATGCTCTCATCAGG-3';

- IL-6, sentido 5'-GCTACCAAACTGGATATAATCAGGA-3' y antisentido 5'-CCAGGTAGCTATGGTACTCCAGAA-3';

- IL-10; sentido 5'-CAGAGCCACATGCTCCTAGA-3' y antisentido 5'-TGTCCAGCTGGTCCTTTGTT-3';

- TNF- $\alpha$ sentido 5'-CAGCCTCTTCTCCTTCCTGAT-3' y antisentido 5'-GCCAGAGGGCTGATTAGAGA-3';

- y para el housekeeping, GAPDH, sentido 5'-GACTAACCCTGCGCTCCTG-3' y antisentido 5'-GCCCAATACGACCAAATCAG-3'.

La reacción de qPCR se inició con un precalentamiento a $90^{\circ} \mathrm{C}$ durante 10 minutos, seguida de 35 ciclos de amplificación (desnaturalización por 60 segundos a $94^{\circ} \mathrm{C}$; alineamiento a $60^{\circ} \mathrm{C}$ durante 10 segundos y extensión a $72^{\circ} \mathrm{C}$ durante 60232 segundos). La expresión relativa se calculó empleando el método $2^{-\Delta \Delta \mathrm{CT}}[16]$.

\section{Análisis estadístico}

Los valores expresados representan la media \pm error estándar (EE) de una n=3 de cada grupo. El análisis de comparación de medias se realizó por ANOVA con un post hoc Bonferroni. Se considero el valor de $\mathrm{p}<0.05$ como significancia estadística.

\section{RESULTADOS Y DISCUSIÓN}

\section{Diferenciación de células THP-1 a macrófagos}

Las células THP-1, se diferenciaron a macrófagos utilizando forbol-12-miristato-13-acetato (PMA). Se sembraron 16x104 células por pozo en placas de 48 pozos y se agregó a cada uno PMA a una concentración de 60 ng/ml. Posterior a 48h, se cambió el medio a las células y se dejaron durante $72 \mathrm{~h}$ con medio fresco (DEMEM/ F12 + 10\% SFB). La diferenciación se confirmó al observar a un aumento 40X que las células se encontraban adheridas a la placa, aumento en la granularidad de la célula y se observaron cambios en la morfología, ya que las células diferenciadas presentan una membrana con bordes irregulares en comparación con las células no diferenciadas que son totalmente esféricas y que además se encuentran en suspensión (Figura 1) ${ }^{[17]}$.

\section{Disminución de la actividad NADPH oxidasa por la acción de tibolona}

Los macrófagos son células del sistema inmune que participan en el proceso inflamatorio y entre sus funciones principales se encuentra la fagocitosis, actividad que consiste en la eliminación de microorganismos, principalmente a través de la activación de un complejo enzimático conocido como NADPH oxidasa que promueve la producción de ERO y ERN ${ }^{[18]}$. La activación se caracteriza por un aumento en el consumo de oxígeno molecular y tiene como objetivo la liberación del radi- 


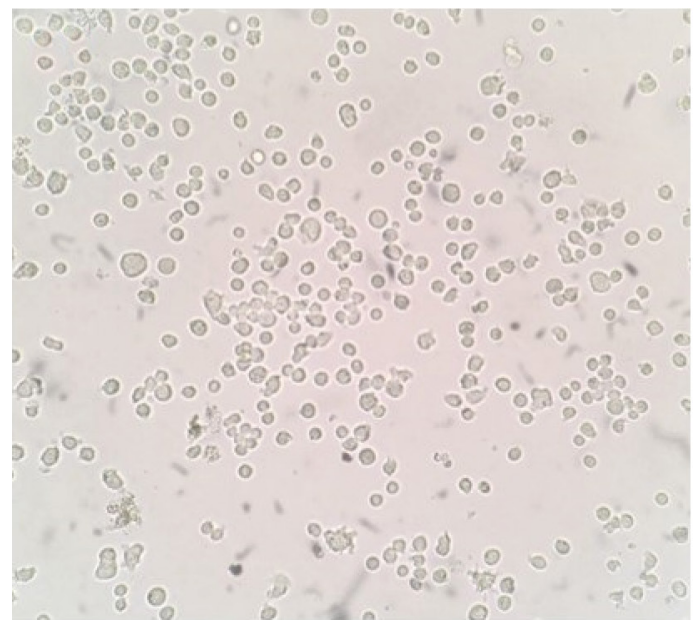

a)

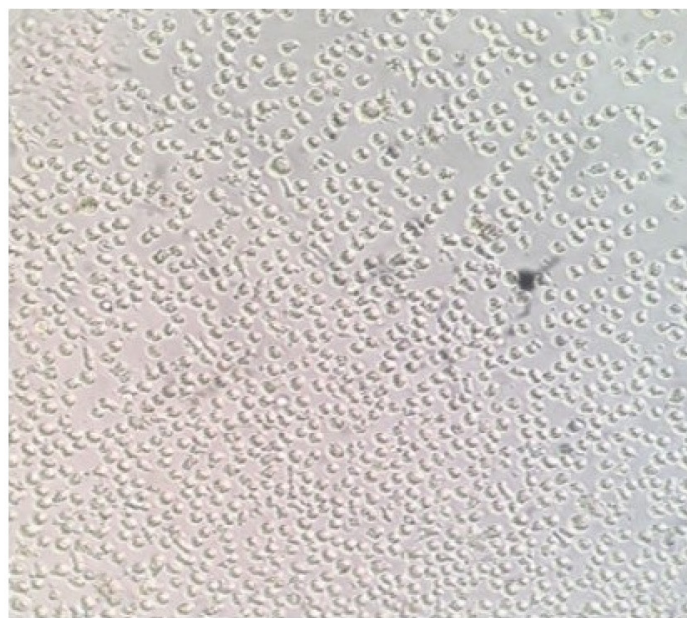

b)

FIGURA 1. Diferenciación de células THP-1 a macrófagos. A) células sin diferenciar y B) células diferenciadas. (40x)

cal anión superóxido dentro de la vacuola fagocítica o al medio extracelular utilizando como sustrato a NADPH ${ }^{[19] . ~ A u n q u e ~ e s t e ~ s i s t e m a ~ e s ~ r e l e v a n t e ~ c o m o ~}$ mecanismo de defensa del organismo, la desregulación de la actividad de la NADPH oxidasa desempeña un papel importante en el desarrollo de diversas enfermedades, particularmente las asociadas con estrés oxidativo como las enfermedades cardiovasculares ${ }^{[20]}$. Tb es un esteroide sintético prescrito para el tratamiento de los síntomas vasomotores y osteoporosis en la mujer en posmenopausia, actúa como regulador de la actividad estrogénica tejido específico, que además ha demostrado tener efectos antiaterogénicos ${ }^{[14]}$.

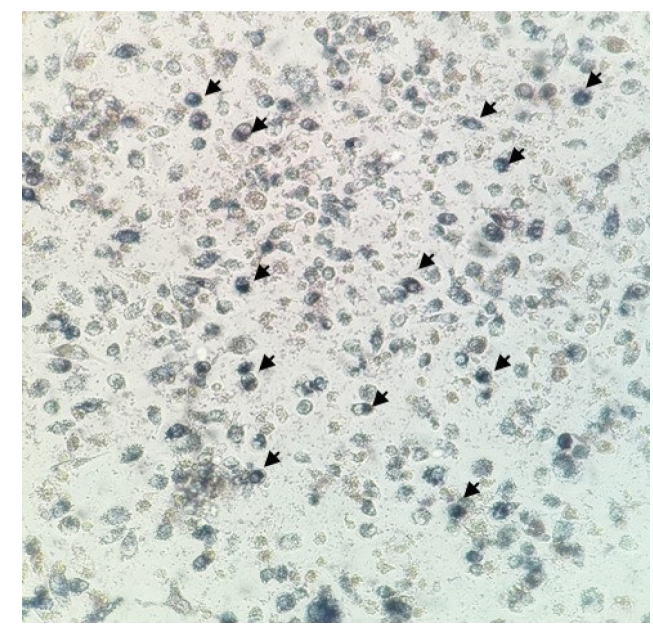

FIGURA 2. Actividad de NADPH oxidada

(con flechas se señalan los cristales). (40x)
La actividad de NADPH oxidasa fue evaluada a través del ensayo con NBT (Figura 2), para lo cual se sembraron $8 \times 10^{4} \mathrm{cel} /$ pozo en una placa de 96 pozos.

En el grupo control positivo $\operatorname{Ctrl}(+)$, se observó un incremento en la actividad de NADPH oxidasa, con respecto al grupo control negativo $\mathrm{Ctrl}(-)$, a las $24 \mathrm{~h}(\mathrm{p}<0.05)$ y 48h ( $<<0.01)$, lo cual indica que el modelo de activación enzimática con PMA (100 ng/ml) es adecuado para estudiar efectos sobre este complejo (Figura 3 y 4).

En este estudio se observó que Tb mantiene la actividad enzimática similar a la obtenida en el grupo Ctrl (-), aún después de que las células han recibido estímulo con PMA, el cual es un activador del complejo enzimático antes mencionado ${ }^{[21]}$ y no se encontró diferencia estadísticamente significativa entre el grupo Ctrl (-) y los grupos que se preincubaron con $\mathrm{Tb}$ a una concentración de $10 \mu \mathrm{M}$. Sin embargo, si se evidenció una diferencia significativa cuando se compararon los grupos con tratamiento con el grupo Ctrl (+) a las $24 \mathrm{~h}$ y $48 \mathrm{~h}(\mathrm{p}<0.001$ ) (Figura 3 ). Lo cual sugiere que Tb regula la actividad del complejo enzimático NADPH oxidasa, posiblemente a través de la modulación del efecto estrogénico de sus metabolitos, lo cual concuerda con los estudios que reportaron una disminución de la actividad enzimática por los estrógenos ${ }^{[7]}$. 


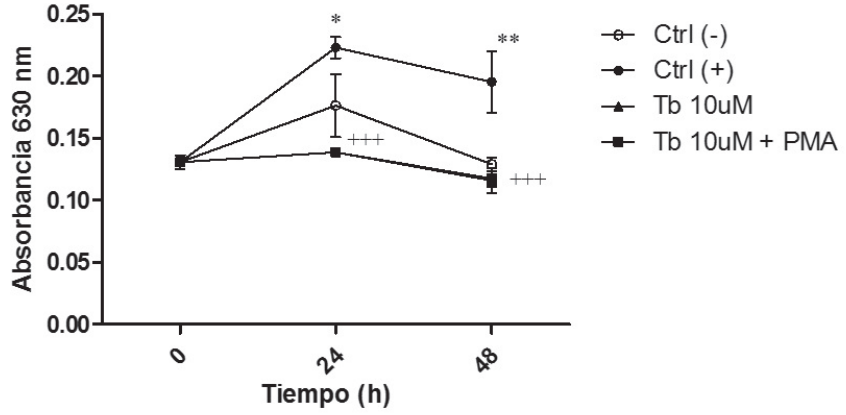

FIGURA 3. Disminución de la actividad NADPH oxidasa en macrófagos preincubados con tibolona. Los signos * y + indican diferencia estadísticamente significativa con respecto al grupo Ctrl (-) y al Ctrl (+), respectivamente.

En los grupos preincubados con $\mathrm{Tb}$ a una concentración de $1 \mu \mathrm{M}$, la diferencia con respecto al grupo Ctrl (+) sólo se observó a las $24 \mathrm{~h}$, lo cual sugiere que el efecto del fármaco en la regulación de la actividad NADPH oxidasa es dependiente de la concentración del fármaco (Figura 4).

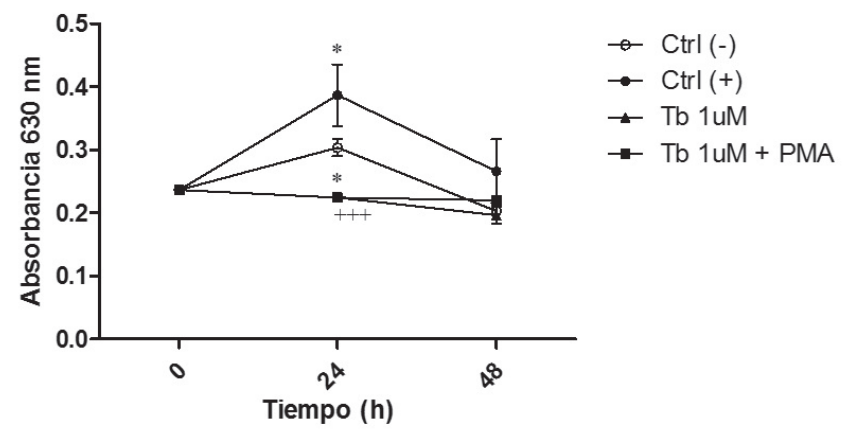

FIGURA 4. Disminución de la actividad NADPH oxidasa en macrófagos preincubados con tibolona. Los signos * y + indican diferencia estadísticamente significativa con respecto al grupo Ctrl (-) y al Ctrl (+), respectivamente.

\section{Cambios en la expresión génica de citocinas en el macrófago}

Estudios en líneas celulares de macrófagos han demostrado el efecto regulador antiinflamatorio de los estrógenos vía activación de su receptor ${ }^{[22,23]}$, por lo que en este estudio se evaluó el efecto de Tb, un fármaco que actúa a través de la activación de los receptores de ER, en la actividad del macrófago, para lo cual se determinó la expresión de citocinas (IL-1ß, IL-6, TNF- $\alpha$ e IL-10) a través de la técnica de RT-qPCR en macrófagos preincubados con Tb 10 y $1 \mu \mathrm{M}$ durante 24h. Para realizar este ensayo se sembraron 1x105 cel/ pozo en placas de 6 pozos. Los resultados de este estudio muestran que $\mathrm{Tb}$ tiene un efecto en la expresión de las citocinas en el macrófago y que ésta es dependiente de la concentración del fármaco.

En relación a la interleucina 1, se encontró que el grupo incubado con Tb-PMA 10uM hubo menor expresión de IL-1, con respecto al control en el tiempo o (estado basal), mientras que en el grupo con Tb a una concentración de $1 \mathrm{uM}$, se mantiene sin cambios. Por otro lado, no se encontró diferencia estadísticamente significativa entre el grupo que solo se incubo con $\mathrm{Tb}$ (10uM) y el grupo que además del tratamiento a la misma concentración recibió el estímulo. Sin embargo, en el grupo que se preincubó con Tb 1uM y además recibió el estímulo con PMA, hubo menor expresión de IL-1, con respecto al grupo que sólo se incubó con el tratamiento (Figura 5).

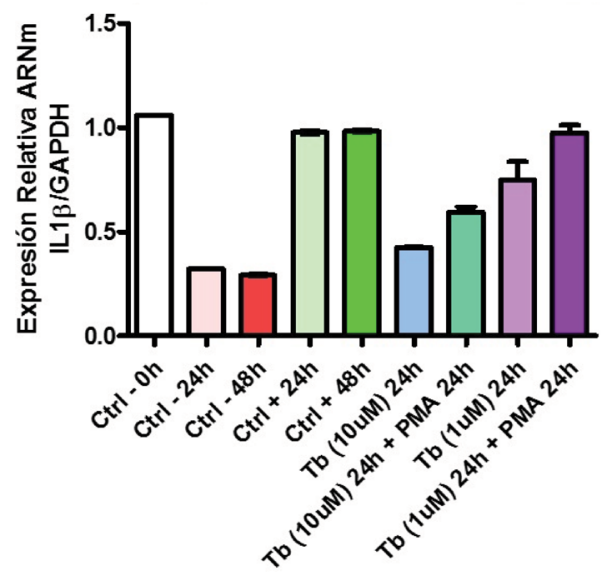

FIGURA 5. Expresión de IL-1 $\beta$ en macrófagos preincubados con tibolona.

En cuanto a la expresión de IL-6, en el grupo incubado solo con Tb (1uM) se observó mayor expresión de IL-6 con respecto al grupo Ctrl 0, sin encontrar cambios en el grupo con Tb 10uM. 
Pero cuando Tb 1uM se preincubó con PMA, se observó menor expresión de esta citocina, en comparación con el grupo que sólo se preincubó con Tb (1uM). Sin embargo, no se encontró diferencia estadísticamente significativa con respecto a la expresión de IL-6 en los grupos con y sin estimulo, preincubado con Tb 10uM (Figura 6).

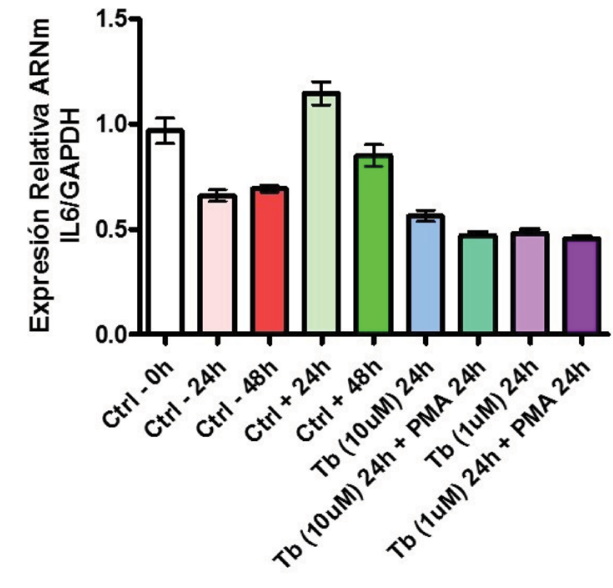

FIGURA 6. Expresión de IL-6 en macrófagos preincubados con tibolona.

Se observó que hubo mayor expresión de TNF- $\alpha$ en el grupo preincubado con $\mathrm{Tb} 10 \mathrm{uM}$, con respecto al grupo preincubado con Tb más PMA. El mismo efecto se observó en los grupos que fueron tratados con $\mathrm{Tb}$ 1uM, con y sin estimulo (Figura 7).

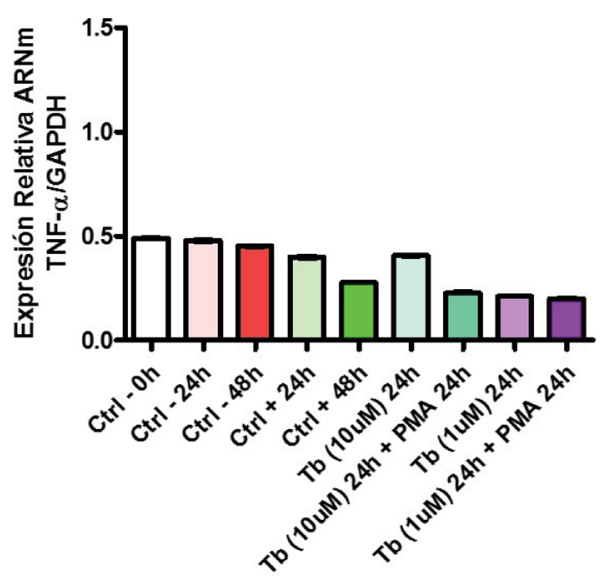

FIGURA 7. Expresión de TNF- $\alpha$ en macrófagos preincubados con tibolona.
Con respecto a la expresión de IL-10, se observó que en el grupo incubado con Tb 10uM, hubo menor expresión de la citocina con respecto al grupo Ctrl 0 , mientras que en el grupo con la concentración menor (1uM), presentó mayor expresión de IL-10. Además, se observó que en el grupo tratado con Tb 10uM la expresión de IL-10 fue menor en comparación con el grupo que recibió el tratamiento y el estímulo. Sin embargo, en el grupo tratado con Tb 1uM, el efecto observado fue contrario al que recibió tanto tratamiento como estímulo con PMA, ya que hubo mayor expresión de IL-10 (Figura 8).

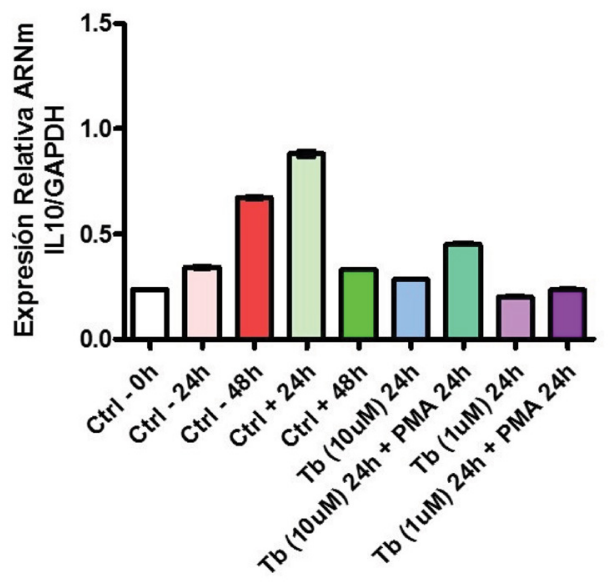

FIGURA 8. Figura 8. Expresión de IL-10 en macrófagos preincubados con tibolona.

Existen estudios in vitro con células mononucleares obtenidas de sangre periférica de pacientes en posmenopausia en tratamiento con $\mathrm{Tb}$, en donde no se han encontrado cambios en los niveles séricos de algunas citocinas ${ }^{[24]}$, en este estudio (in vitro) con macrófagos, aunque no se encontraron cambios en la expresión de IL-6, si se observó menor expresión citocinas proinflamatorias (IL-1 $\beta$ y TNF- $\alpha$ ), efecto observado también en un modelo animal (in vivo) en donde se redujeron las concentraciones séricas de TNF- $\alpha$ en grupos tratados con $\mathrm{Tb}^{[25]}$. Estos resultados sugieren que $\mathrm{Tb}$ tiene una actividad moduladora de la síntesis de citocinas, por lo que podría jugar un papel importante en la determinación del perfil de los macrófagos. 
El presente estudio demostró que Tb tiene un efecto regulador de la actividad enzimática NADPH oxidasa del macrófago, lo cual podría favorecer la disminución de la producción de especies reactivas de Nitrógeno y Oxigeno, producto de la activación de este complejo enzimático. Asimismo, se demostró que Tb también tiene efectos moduladores en la síntesis de citocinas (IL-1 $\beta$, IL-6, TNF- $\alpha$ y IL-10); posiblemente como consecuencia de su actividad sobre el receptor de estrógenos, que se ha demostrado está involucrado en la regulación de la síntesis y expresión de citocinas a través de la vía NF- $\kappa$ B. Los resultados contribuyen a la descripción de la actividad de este fármaco, además se pro- pone al macrófago como un posible blanco terapéutico en la regulación del proceso inflamatorio por la acción de Tb. Por lo que, se sugiere realizar la cuantificación de proteínas asociadas a las citocinas, así como la evaluación de la síntasa del óxido nítrico inducible (iSON)un complejo enzimático también involucrado en la producción de especies reactivas.

\section{CONCLUSIONES}

Tibolona es un fármaco que favorece el fenotipo antiinflamatorio de los macrófagos a través de la modulación de la actividad enzimática celular, así como en la expresión génica de citocinas. 


\section{REFERENCIAS}

[1] Barañao, R.I., Hormonas sexuales y respuesta inmunológica. Revista de la Sociedad Argentina de Endocrinología Ginecológica y Reproductiva, 2009. 16: p. 20-30. URL: http://hdl.handle. net/11336/8776

[2] Fujiwara, N. and K. Kobayashi, Macrophages in inflammation. Current Drug Targets-Inflammation \& Allergy, 2005. 4(3): p. 281286. doi.org/10.2174/1568010054022024

[3] Bastarrachea, R.A., et al., [Macrophages, inflammation, adipose tissue, obesity and insulin resistance]. Gac Med Mex, 2007. 143(6): p. 505-12. PMID: 18269082

[4] Toniolo, A., et al., Alternative activation of human macrophages is rescued by estrogen treatment in vitro and impaired by menopausal status. The Journal of Clinical Endocrinology \& Metabolism, 2015. 100(1): p. E50-E58. doi.org/10.1210/ic.2014-2751

[5] Ma, J., et al., Regulation of macrophage activation. Cellular and Molecular Life Sciences CMLS, 2003. 60(11): p. 2334-2346. doi.org/10.1007/s00018-003-3020-0

[6] Mantovani, A., et al., The chemokine system in diverse forms of macrophage activation and polarization. Trends in immunology, 2004. 25(12): p. 677-686. doi.org/10.1016/j.it.2004.09.015

[7] Sumi, D., et al., 17ß-Estradiol inhibits NADPH oxidase activity through the regulation of p47phox mRNA and protein expression in THP-1 cells. Biochimica et Biophysica Acta (BBA)-Molecular Cell Research, 2003. 1640(2-3): p. 113-118. doi.org/10.1016/S0167-4889(03)00026-0

[8] Vassalle, C., et al., Effects of menopause and tibolone on different cardiovascular biomarkers in healthy women. Gynecological Endocrinology, 2011. 27(3): p. 163-169. doi.org/10.3109/09513590.2010.488770

[9] Skouby, S.O., et al., The effect of continuous combined conjugated equine estrogen plus medroxyprogesterone acetate and tibolone on cardiovascular metabolic risk factors. Climacteric, 2008. 11(6): p. 489-497. doi.org/10.1080/13697130802455150

[10] Koh, K.K., et al., Significant differential effects of hormone therapy or tibolone on markers of cardiovascular disease in postmenopausal women: a randomized, double-blind, placebocontrolled, crossover study. Arteriosclerosis, thrombosis, and vascular biology, 2003. 23(10): p. 1889-1894. doi.org/10.1161/01.ATV.0000091502.96745. 95

[11] Gompel, A., et al., Tibolone actions on normal and breast cancer cells. European Journal of Cancer, 2000. 36: p. 76-77. doi.org/10.1016/50959-8049(00)00236-7

[12] Simoncini, T., et al., Tibolone activates nitric oxide synthesis in human endothelial cells. The Journal of Clinical Endocrinology \& Metabolism, 2004. 89(9): p. 4594-4600. doi.org/10.1210/jc.2003-032189

[13] Cicinelli, E., et al., Effects of tibolone on plasma levels of nitric oxide in postmenopausal women. Fertility and sterility, 2002. 78(3): p. 464-468. doi.org/10.1016/S0015-0282(02)03295-8
[14] Simoncini, T. and A. Genazzani, Tibolone inhibits leukocyte adhesion molecule expression in human endothelial cells. Molecular and cellular endocrinology, 2000. 162(1-2): p. 87-94. doi.org/10.1016/50303-7207(00)00206-9

[15] Blok, L., et al., Progestagenic effects of tibolone on human endometrial cancer cells. The Journal of Clinical Endocrinology \& Metabolism, 2003. 88(5): p. 2327-2334. doi.org/10.1210/jc.2002-021737

[16] Livak, K. J., \& Schmittgen, T. D. (2001). Analysis of relative gene expression data using real-time quantitative PCR and the $2-\Delta \Delta \mathrm{CT}$ method. methods, 25(4), 402-408. doi.org/10.1006/meth.2001.1262

[17] Daigneault, M., et al., The identification of markers of macrophage differentiation in PMA-stimulated THP-1 cells and monocytederived macrophages. PloS one, 2010. 5(1): p. e8668. doi.org/10.1371/journal.pone.0008668

[18] Arango Rincón, J.C., L.Y. Gámez Díaz, and J.Á. López Quintero, NADPH oxidase system: new challenges and perspectives. Iatreia, 2010. 23(4): p. 362-372. ISSN: 0121-0793

[98] Vazquez-Torres, A., et al., Antimicrobial actions of the NADPH phagocyte oxidase and inducible nitric oxide synthase in experimental salmonellosis. I. Effects on microbial killing by activated peritoneal macrophages in vitro. Journal of Experimental Medicine, 2000. 192(2): p. 227-236. doi.org/10.1084/iem.192.2.227

[20] Cathcart, M.K., Regulation of superoxide anion production by NADPH oxidase in monocytes/macrophages: contributions to atherosclerosis. Arteriosclerosis, thrombosis, and vascular biology, 2004. 24(1): p. 23-28. doi.org/10.1161/01.ATV.0000097769.47306.12

[21] Juliet, P.A., et al., Combined effect of testosterone and apocynin on nitric oxide and superoxide production in PMA-differentiated THP-1 cells. Biochimica et Biophysica Acta (BBA)-Molecular Cell Research, 2004. 1693(3): p. 185-191. doi.org/10.1016/j.bbamcr.2004.08.002

[22] Villa, A., et al., Estrogen accelerates the resolution of inflammation in macrophagic cells. Sci Rep, 2015. 5: p. 15224. doi.org/10.1038/srep15224

[23] Kramer, P. and S. Wray, 17- $\beta$-Estradiol regulates expression of genes that function in macrophage activation and cholesterol homeostasis. The Journal of steroid biochemistry and molecular biology, 2002. 81(3): p. 203-216. doi.org/10.1016/50960-0760(02)00065-1

[24] Vitale, C., et al., Interleukin-6 and flow-mediated dilatation as markers of increased vascular inflammation in women receiving hormone therapy. Menopause, 2005. 12(5): p. 552-558. doi.org/10.1097/01.gme.0000172267.24949.70

[25] de Medeiros, A.R.S., et al., Tibolone has anti-inflammatory effects in estrogen-deficient female rats on the natriuretic peptide system and TNF-alpha. Regulatory peptides, 2012. 179(1-3): p. 55-60. doi.org/10.1016/j.regpep.2012.08.015 